\title{
Steps toward Determination of the Size and Structure of the Broad\#Line Region in Active Galactic Nuclei. IX. Ultraviolet Observations of Fairall 9
}

\section{Citation}

Rodriguez\#Pascual, P. M., D. Alloin, J. Clavel, D. M. Crenshaw, K. Horne, G. A. Kriss, J. H. Krolik, et al. 1997. "Steps Toward Determination of the Size and Structure of the Broad\#Line Region in Active Galactic Nuclei. IX. Ultraviolet Observations of Fairall 9." The Astrophysical Journal Supplement Series 110 (1) (May): 9-20. doi:10.1086/312996.

\section{Published Version}

doi:10.1086/312996

\section{Permanent link}

http://nrs.harvard.edu/urn-3:HUL.InstRepos:30212162

\section{Terms of Use}

This article was downloaded from Harvard University's DASH repository, and is made available under the terms and conditions applicable to Other Posted Material, as set forth at http:// nrs.harvard.edu/urn-3:HUL.InstRepos:dash.current.terms-of-use\#LAA

\section{Share Your Story}

The Harvard community has made this article openly available.

Please share how this access benefits you. Submit a story. 
The Astrophysical Journal SuPPLEMENT SerIes, 110:9-20, 1997 May

(C) 1997. The American Astronomical Society. All rights reserved. Printed in U.S.A.

\title{
STEPS TOWARD DETERMINATION OF THE SIZE AND STRUCTURE OF THE BROAD-LINE REGION IN ACTIVE GALACTIC NUCLEI. IX. ULTRAVIOLET OBSERVATIONS OF FAIRALL 9
}

\author{
P. M. Rodríguez-Pascual, ${ }^{1}$ D. Alloin, ${ }^{2}$ J. Clavel,${ }^{3}$ D. M. Crenshaw, ${ }^{4}$ K. Horne, ${ }^{5}$ G. A. Kriss, ${ }^{6}$ J. H. Krolik, ${ }^{6}$ \\ M. A. Malkan, ${ }^{7}$ H. Netzer, ${ }^{8}$ P. T. O’Brien, ${ }^{9}$ B. M. Peterson, ${ }^{10}$ G. A. Reichert, ${ }^{11}$ W. Wamsteker, ${ }^{1}$ \\ T. AleXANDer, ${ }^{8}$ P. BARr, ${ }^{3}$ R. D. Blandford, ${ }^{12}$ J. N. Bregman, ${ }^{13}$ T. E. CARone, ${ }^{14,15}$ \\ S. Clements, ${ }^{16}$ T.-J. Courvoisier, ${ }^{17}$ M. M. De Robertis, ${ }^{18}$ M. Dietrich, ${ }^{19}$ H. Dottori, ${ }^{20}$ R. A. Edelson, ${ }^{21}$ \\ A. V. Filippenko, ${ }^{22}$ C. M. Gaskell, ${ }^{23}$ J. P. Huchra, ${ }^{24}$ J. B. Hutchings, ${ }^{25}$ W. KollatschnY, ${ }^{26}$ A. P. KoratKar, ${ }^{27}$ \\ K. T. Korista, ${ }^{28}$ A. LAOR, ${ }^{29}$ G. M. MaCAlpine, ${ }^{13}$ P. G. MARTIN, ${ }^{30}$ D. MaOz, ${ }^{8}$ B. McCollum, ${ }^{31}$ S. L. Morris, ${ }^{25}$ \\ G. C. Perola, ${ }^{32}$ R. W. Pogge, ${ }^{10}$ R. L. PtaK, ${ }^{33}$ M. C. Recondo-GonzÁlez, ${ }^{34}$ J. M. Rodríguez-EsPinoza, ${ }^{35}$ \\ E. L. Rokaki, ${ }^{36}$ M. Santos-Lleó, ${ }^{37}$ K. SeKiguchi, ${ }^{38}$ J. M. Shull, ${ }^{39}$ M. A. J. SniJders, ${ }^{40}$ L. S. Sparke, ${ }^{41}$ \\ G. M. Stirpe, ${ }^{42}$ R. E. Stoner, ${ }^{33}$ W.-H. Sun, ${ }^{43}$ S. J. WAgner, ${ }^{19}$ I. Wanders, ${ }^{10}$ \\ B. J. WILKES, ${ }^{24}$ C. WINGE, ${ }^{20,27}$ AND W. ZHENG ${ }^{6}$ \\ Received 1996 October 24; accepted 1996 December 16
}

\begin{abstract}
An 8 month monitoring campaign on the Seyfert 1 galaxy Fairall 9 has been conducted with the International Ultraviolet Explorer in an attempt to obtain reliable estimates of continuum-continuum and continuum-emission-line delays for a high-luminosity active galactic nucleus (AGN). While the results of this campaign are more ambiguous than those of previous monitoring campaigns on lower luminosity sources, we find general agreement with the earlier results: (1) there is no measurable lag between ultraviolet continuum bands, and (2) the measured emission-line time lags are very short. It is especially notable that the $\operatorname{Ly} \alpha+\mathrm{N}$ v emission-line lag is about 1 order of magnitude smaller than determined from a previous campaign by Clavel, Wamsteker, \& Glass (1989) when Fairall 9 was in a more luminous state. In other well-monitored sources, specifically NGC 5548 and NGC 3783, the highest ionization lines are found to respond to continuum variations more rapidly than the lower ionization lines, which suggests a radially ionization-stratified broad-line region. In this case, the results are less certain, since none of the emission-line lags are very well determined. The best-determined emission line lag is $\operatorname{Ly} \alpha+\mathrm{N} \mathrm{V}$, for which we find that the centroid of the continuum-emission-line crosscorrelation function is $\tau_{\text {cent }} \approx 14-20$ days. We measure a lag $\tau_{\text {cent }} \lesssim 4$ days for He II $\lambda 1640$; this result is consistent with the ionization-stratification pattern seen in lower luminosity sources, but the relatively large uncertainties in the emission-line lags measured here cannot rule out similar lags for $\mathrm{Ly} \alpha+\mathrm{N} v$ and $\mathrm{He}$ II $\lambda 1640$ at a high level of significance. We are unable to determine a reliable lag for $\mathrm{C}$ IV $\lambda 1550$, but we note that the profiles of the variable parts of Ly $\alpha$ and C IV $\lambda 1550$ are not the same, which does not support the hypothesis that the strongest variations in these two lines arise in the same region.
\end{abstract}

Subject headings: galaxies: active — galaxies: individual (Fairall 9) — galaxies: Seyfert — ultraviolet: galaxies

${ }^{1}$ ESA IUE Observatory, P.O. Box 50727, 28080 Madrid, Spain.

${ }^{2}$ Centre d'Etudes de Saclay, Service d'Astrophysique, Orme des Merisiers, 91191 Gif-sur-Yvette Cedex, France.

${ }^{3}$ ISO Project, European Space Agency, Apartado 50727, 28080 Madrid, Spain.

${ }^{4}$ Computer Sciences Corporation, Laboratory for Astronomy and Solar Physics, NASA/Goddard Space Flight Center, Code 681, Greenbelt, MD 20771.

${ }^{5}$ School of Physics and Astronomy, University of St. Andrews, North Haugh, St. Andrews KY16 9SS, Scotland, UK.

${ }^{6}$ Department of Physics and Astronomy, Johns Hopkins University, Baltimore, MD 21218.

${ }^{7}$ Department of Astronomy, University of California, Math-Science Building, Los Angeles, CA 90024

${ }^{8}$ School of Physics and Astronomy and the Wise Observatory, Raymond and Beverly Sackler Faculty of Exact Sciences, Tel Aviv University, Tel Aviv 69978, Israel.

9 Department of Physics and Astronomy, University of Leicester, University Road, Leicester LE1 7RH, England, UK.

${ }^{10}$ Department of Astronomy, Ohio State University, 174 West 18 th Avenue, Columbus, $\mathrm{OH} 43210$.

${ }_{11}$ NASA/Goddard Space Flight Center, Code 631, Greenbelt, MD 20771.

12 Theoretical Astrophysics, Caltech 130-33, Pasadena, CA 91125.

${ }^{13}$ Department of Astronomy, University of Michigan, Dennison Building, Ann Arbor, MI 48109.

${ }^{14}$ Space Sciences Laboratory, University of California, Berkeley, and Eureka Scientific, Inc.

${ }^{15}$ Present address: 28740 West Fox River Drive, Cary, IL 60013.

16 Department of Astronomy, University of Florida, P.O. Box 112055, 211 SSRB, Gainesville, FL 32611-2055.

${ }^{17}$ Observatoire de Genève, $\mathrm{CH}-1290$ Sauverny, Switzerland.

${ }_{18}$ Department of Physics and Astronomy, York University, 4700 Keele Street, North York, ON M3J 1P3, Canada.

${ }^{19}$ Landessternwarte, Königstuhl, D-69117 Heidelberg, Germany.

${ }^{20}$ Departamento de Astronomia, Instituto de Física, Universidade Federal do Rio Grande do Sul, Avenida Bento Gonçalves, 9500, CP15051, CEP 91500, Porto Alegre, RS, Brazil.

${ }^{21}$ Department of Physics and Astronomy, University of Iowa, Iowa City, IA 52242.

22 Department of Astronomy, University of California, Berkeley, CA 94720-3411.

${ }^{23}$ Department of Physics and Astronomy, University of Nebraska, Lincoln, NE 68588.

${ }^{24}$ Harvard-Smithsonian Center for Astrophysics, 60 Garden Street, Cambridge, MA 02138.

${ }^{25}$ Dominion Astrophysical Observatory, 5071 West Saanich Road, Victoria, B.C. V8X 4M6, Canada. 


\section{INTRODUCTION}

With the discovery of coordinated continuum and emission-line variability in active galactic nuclei (AGNs), it was realized by many authors that variability might provide a very valuable tool for unveiling the structure of the innermost regions of these extremely luminous objects. The rapid response of the emission lines to continuum variations strongly argues that the line emission is driven by photoionization by the AGN central source, which is generally supposed to be an accretion disk surrounding a supermassive black hole. Thus, by measuring the time-delayed response of the emission lines to continuum variations, it is in principle possible to infer the distribution and kinematics of the line-emitting gas through a process known as "reverberation mapping" (e.g., Blandford \& McKee 1982). Multiwavelength measurements of continuum and emission-line variability also provide important observational constraints on the central source itself by providing a probe (1) of the unobservable ionizing continuum, through the response of the emission lines to changes in the observable continuum, and (2) of the structure of the accretion disk itself, since in any model with a radial temperature gradient (such as a thin accretion disk), different continuum wavelengths arise principally at different disk radii.

On account of the rapid and irregular nature of the continuum variations, it was also realized early that enormous observational efforts were needed to exploit the potential of variability studies. The techniques to obtain the necessary information (reviewed in Gondhalekar, Horne, \& Peterson 1994) require long series of high-quality spectroscopic data, preferably taken at nearly regular intervals. The International Ultraviolet Explorer (IUE) is extremely well suited to this task, which motivated the establishment of international consortia in the late 1980 s to conduct a series of major observing campaigns using both $I U E$ and ground-based facilities to obtain the necessary spectroscopic observations. The largest of these groups, the International AGN Watch (Alloin et al. 1994), has now completed multiwavelength programs on NGC 5548 (Clavel et al. 1991, [Paper I in this series]; Peterson et al. 1991 [Paper II], 1992 [Paper III], 1994 [Paper VII]; Dietrich et al. 1993 [Paper IV]; Maoz et al. 1993; Romanishin et al. 1995; Korista et al. 1995 [Paper VIII]), NGC 3783 (Reichert et al. 1994 [Paper V]; Stirpe et al. 1994 [Paper VI]; Alloin et al. 1995), and NGC 4151 (Crenshaw et al. 1996; Kaspi et al. 1996; Warwick et al. 1996; Edelson et al. 1996). The general results of these AGN monitoring campaigns can be summarized as follows:

1. The UV and optical continua vary simultaneously within the accuracy of the data and the sampling frequency (typically \pm 2 days or less).

2. The emission-line lags (or mean response times to continuum variations) are found to be quite small, typically ranging between a few days to several weeks.

3. The high-ionization lines (e.g., $\mathrm{N}$ v $\lambda 1240$, He II $\lambda 1640$, and $\mathrm{C}$ IV 21550 ) respond faster to continuum changes than the low-ionization lines (e.g., $\mathrm{Mg}$ II $\lambda 2800, \mathrm{H} \beta$ ).

The luminosity range for which these results have been obtained $\left[10^{39} \lesssim L_{\lambda}(1450 \AA) \lesssim 10^{40} \mathrm{ergs} \mathrm{s}^{-1} \AA^{-1}\right]$ is still too small to test critically the expected $R \propto L^{1 / 2}$ relationship between the broad-line region (BLR) size $R$ and continuum luminosity $L$ predicted by simple photoionization equilibrium arguments (e.g., Peterson 1993), and extension of variability studies to higher luminosity is therefore necessary. In this regard, the luminous Seyfert galaxy Fairall 9 $\left[L_{\lambda}(1450 \AA)>10^{41} \operatorname{ergs~s}^{-1} \AA^{-1} ; z=0.047\right]$ is of interest, as it is one of the few high-luminosity sources that have been monitored with IUE. IUE observations with an average sampling interval of about 90 days suggested emission-line lags of about $115 \pm 70$ days for $\operatorname{Ly} \alpha$ and about $200 \pm 80$ days for C IV $\lambda 1550$ (Clavel, Wamsteker, \& Glass 1989; see also Koratkar \& Gaskell 1989; Lub \& de Ruiter 1992; Shields, Ferland, \& Peterson 1995; RecondoGonzález et al. 1997) relative to the UV continuum during a time when Fairall 9 underwent a dramatic change in luminosity. Between 1978 and 1985, the UV flux of Fairall 9 decreased nearly monotonically by a factor of about 30 .

In view of its well-documented UV variability history and its importance in the $R-L$ relationship, we decided to undertake a more intensive monitoring program on Fairall 9. Our goal was to obtain observations with the same sampling frequency (about one observation every 4 days) and dura-

\footnotetext{
26 Universitäts-Sternwarte Göttingen, Geismarlandstrasse 11, D-37083 Göttingen, Germany.

27 Space Telescope Science Institute, 3700 San Martin Drive, Baltimore, MD 21218.

28 Department of Physics and Astronomy, University of Kentucky, Lexington, KY 40506.

29 Physics Department, Technion Israel Institute of Technology, Haifa 32000, Israel.

${ }^{30}$ Canadian Institute for Theoretical Astrophysics, University of Toronto, Toronto, ON M5S 3H8, Canada.

31 Computer Sciences Corporation, NASA/Goddard Space Flight Center, Code 684.9, Greenbelt, MD 20771.

32 Istituto Astronomico dell'Università, Via Lancisi 29, I-00161 Rome, Italy.

33 Department of Physics and Astronomy, Bowling Green State University, Bowling Green, OH 43403.

${ }^{34}$ Facultad de Ciencias, Departament de Físicas, Universidad de Oviedo, C/ Calvo Sotelo, s/n. Oviedo, Asturias, Spain.

35 Instituto de Astrofísica de Canarias, E-38200 La Laguna, Tenerife, Spain.

36 Royal Observatory Edinburgh, University of Edinburgh, Blackford Hill, Edinburgh, EH9 3HJ, Scotland, UK.

37 LAEFF, Apartado 50727, E-28080 Madrid, Spain.

38 South African Astronomical Observatory, P.O. Box 9, Observatory 7935, South Africa.

39 Joint Institute for Laboratory Astrophysics, University of Colorado and National Institute of Standards and Technology, Campus Box 440, Boulder, CO 80309.

40 IRAM, 300 Rue de la Piscine, 38046 Saint Martin d'Heres, France.

${ }^{41}$ Department of Astronomy, University of Wisconsin, 475 North Charter Street, Madison, WI 53706

42 Osservatorio Astronomico di Bologna, Via Zamboni 33, I-40126, Bologna, Italy.

${ }^{43}$ Institute of Astronomy, National Central University, Chung-Li, Taiwan 32054, Republic of China.
} 
TABLE 1

LOG OF OBSERVATIONS

\begin{tabular}{|c|c|c|c|c|c|}
\hline $\begin{array}{c}\text { SWP } \\
\text { Image Number } \\
\text { (1) }\end{array}$ & $\begin{array}{l}\text { UT } \\
\text { Date } \\
(2)\end{array}$ & $\begin{array}{c}\text { UT Start } \\
(\mathrm{hh}: \mathrm{mm}: \mathrm{ss}) \\
(3)\end{array}$ & $\begin{array}{c}\text { JD } \\
(-2,449,000) \\
(4)\end{array}$ & $\begin{array}{l}\text { Exposure Time } \\
\text { (minutes) } \\
\text { (5) }\end{array}$ & $\begin{array}{l}\text { Number of } \\
\text { Segments } \\
\text { (6) }\end{array}$ \\
\hline $50641^{a}$. & 1994 Apr 28 & $04: 26: 11$ & 470.684 & 140 & 7 \\
\hline $50671 \ldots$ & 1994 May 01 & $03: 37: 45$ & 473.652 & 110 & 5 \\
\hline $50700 \ldots \ldots \ldots$ & 1994 May 05 & $04: 26: 31$ & 477.684 & 100 & 6 \\
\hline $50731 \ldots \ldots \ldots$ & 1994 May 10 & 04:07:40 & 482.672 & 116 & 6 \\
\hline $50762 \ldots \ldots \ldots$ & 1994 May 14 & $04: 15: 34$ & 486.676 & 125 & 5 \\
\hline $50807 \quad \ldots \ldots \ldots$ & 1994 May 18 & $04: 15: 26$ & 490.676 & 130 & 5 \\
\hline $50860 \ldots$ & 1994 Мау 22 & $04: 40: 16$ & 494.695 & 105 & 4 \\
\hline $50898 \ldots \ldots \ldots$ & 1994 May 25 & $04: 28: 58$ & 497.688 & 115 & 4 \\
\hline $50936 \ldots \ldots \ldots$ & 1994 Мау 29 & $04: 37: 18$ & 501.691 & 103 & 5 \\
\hline $50994 \quad \ldots \ldots \ldots \ldots$ & 1994 Jun 03 & $10: 01: 08$ & 506.918 & 90 & 5 \\
\hline $51034 \ldots \ldots \ldots$ & 1994 Jun 07 & $09: 52: 11$ & 510.910 & 120 & 6 \\
\hline $51068 \ldots \ldots \ldots$ & 1994 Jun 11 & $10: 33: 10$ & 514.941 & 100 & 5 \\
\hline $51101 \ldots \ldots \ldots$ & 1994 Jun 15 & $05: 56: 38$ & 518.746 & 90 & 5 \\
\hline $51118 \ldots \ldots \ldots$ & 1994 Jun 18 & $05: 55: 23$ & 521.746 & 80 & 4 \\
\hline $51119 \ldots \ldots \ldots$ & 1994 Jun 18 & $08: 02: 33$ & 521.836 & 80 & 4 \\
\hline $51120 \ldots$ & 1994 Jun 18 & $10: 17: 51$ & 521.930 & 70 & 3 \\
\hline $51129 \quad \ldots \ldots \ldots$ & 1994 Jun 19 & $06: 02: 21$ & 522.750 & 80 & 4 \\
\hline $51142 \ldots \ldots \ldots$ & 1994 Jun 20 & 09:04:24 & 523.879 & 80 & 3 \\
\hline $51143 \ldots \ldots \ldots$ & 1994 Jun 20 & $11: 05: 39$ & 523.961 & 80 & 3 \\
\hline $51163 \ldots \ldots \ldots$ & 1994 Jun 22 & $22: 22: 29$ & 526.434 & 130 & 5 \\
\hline $51236 \ldots \ldots \ldots$ & 1994 Jun 26 & $22: 33: 21$ & 530.441 & 115 & 5 \\
\hline $51271 \ldots \ldots \ldots$ & 1994 Jun 30 & 02:08:09 & 533.590 & 92 & 4 \\
\hline $51317 \ldots \ldots \ldots$ & 1994 Jul 04 & $00: 30: 41$ & 537.520 & 85 & 4 \\
\hline $51375 \ldots \ldots \ldots$ & 1994 Jul 09 & $00: 24: 16$ & 542.516 & 110 & 5 \\
\hline $51418 \ldots \ldots \ldots$ & 1994 Jul 13 & $01: 04: 46$ & 546.543 & 87 & 3 \\
\hline $51485 \ldots \ldots \ldots$ & $1994 \mathrm{Jul} 16$ & $23: 56: 50$ & 550.496 & 115 & 4 \\
\hline $51546^{\mathrm{a}} \ldots$ & 1994 Jul 21 & $00: 11: 04$ & 554.508 & 93 & 4 \\
\hline $51596^{\mathrm{a}} \ldots$ & 1994 Jul 25 & $05: 44: 51$ & 558.738 & 85 & 5 \\
\hline $51636 \ldots \ldots \ldots$ & 1994 Jul 28 & $05: 52: 08$ & 561.746 & 85 & 4 \\
\hline $51637 \ldots \ldots \ldots$ & 1994 Jul 28 & $08: 11: 03$ & 561.840 & 85 & 4 \\
\hline $51677 \ldots \ldots \ldots$ & 1994 Aug 01 & $05: 37: 08$ & 565.734 & 85 & 4 \\
\hline $51678 \ldots \ldots \ldots$ & 1994 Aug 01 & $07: 47: 03$ & 565.824 & 60 & 3 \\
\hline $51717^{\mathrm{a}, \mathrm{b}} \ldots \ldots \ldots$ & 1994 Aug 05 & $05: 08: 57$ & 569.715 & 90 & 1 \\
\hline $51741^{\mathrm{b}} \ldots \ldots \ldots$ & 1994 Aug 08 & 02:08:09 & 572.590 & 90 & 1 \\
\hline $51742^{\mathrm{b}}$ & 1994 Aug 08 & $03: 59: 47$ & 572.664 & 50 & 1 \\
\hline $51792^{\mathrm{a}, \mathrm{b}} \ldots \ldots \ldots$ & 1994 Aug 13 & $01: 46: 10$ & 577.574 & 90 & 1 \\
\hline $51793^{\mathrm{b}} \ldots$ & 1994 Aug 13 & $03: 45: 50$ & 577.656 & 75 & 1 \\
\hline $51832^{\mathrm{b}} \ldots$ & 1994 Aug 17 & $01: 45: 48$ & 581.574 & 86 & 1 \\
\hline $51833^{\mathrm{b}} \ldots \ldots \ldots$ & 1994 Aug 17 & $03: 36: 05$ & 581.648 & 70 & 1 \\
\hline $51861^{\mathrm{b}}$ & 1994 Aug 21 & $01: 49: 12$ & 585.574 & 85 & 1 \\
\hline $51862^{\mathrm{b}}$ & 1994 Aug 21 & $03: 48: 34$ & 585.660 & 75 & 1 \\
\hline $51911 \ldots \ldots \ldots$ & 1994 Aug 24 & $22: 07: 08$ & 589.422 & 90 & 4 \\
\hline $51973 \ldots \ldots \ldots$ & 1994 Aug 28 & $21: 50: 07$ & 593.410 & 130 & 7 \\
\hline $51998 \ldots \ldots \ldots$ & 1994 Sep 01 & $20: 12: 50$ & 597.340 & 117 & 5 \\
\hline $52039 \ldots \ldots \ldots$ & 1994 Sep 05 & $20: 03: 05$ & 601.336 & 113 & 5 \\
\hline $52072 \ldots \ldots \ldots$ & 1994 Sep 09 & $20: 29: 44$ & 605.352 & 115 & 4 \\
\hline $52106 \ldots \ldots \ldots$ & 1994 Sep 13 & $20: 48: 25$ & 609.367 & 100 & 4 \\
\hline $52142 \ldots \ldots \ldots \ldots$ & $1994 \operatorname{Sep} 17$ & $16: 10: 44$ & 613.172 & 130 & 5 \\
\hline $52174 \ldots \ldots \ldots$ & 1994 Sep 21 & $20: 46: 26$ & 617.367 & 95 & 5 \\
\hline $52194^{a} \ldots \ldots \ldots$ & $1994 \operatorname{Sep} 24$ & $23: 55: 24$ & 620.497 & 35 & 2 \\
\hline $52195 \ldots \ldots \ldots$ & 1994 Sep 25 & 01:03:41 & 620.543 & 90 & 4 \\
\hline $52211 \ldots \ldots \ldots$ & 1994 Sep 25 & $20: 46: 46$ & 621.367 & 100 & 4 \\
\hline $52235 \ldots \ldots \ldots$ & $1994 \operatorname{Sep} 28$ & $03: 45: 56$ & 623.656 & 90 & 4 \\
\hline $52236 \ldots \ldots \ldots$ & 1994 Sep 28 & $06: 13: 53$ & 623.758 & 35 & 1 \\
\hline $52279^{\mathrm{a}} \ldots \ldots \ldots \ldots$ & 1994 Oct 02 & $02: 18: 04$ & 627.596 & 90 & 4 \\
\hline $52343^{\mathrm{a}} \ldots \ldots \ldots \ldots$ & 1994 Oct 06 & $21: 53: 13$ & 632.412 & 95 & 4 \\
\hline $52344 \ldots \ldots \ldots$ & 1994 Oct 07 & $00: 14: 33$ & 632.508 & 60 & 2 \\
\hline $52370^{\mathrm{a}} \ldots \ldots \ldots \ldots$ & 1994 Oct 10 & $21: 56: 02$ & 636.414 & 80 & 4 \\
\hline $52371^{\mathrm{a}} \ldots \ldots \ldots \ldots$ & 1994 Oct 10 & $23: 57: 39$ & 636.498 & 50 & 2 \\
\hline $52406 \ldots \ldots \ldots$ & 1994 Oct 15 & $02: 16: 39$ & 640.594 & 80 & 5 \\
\hline $52516 \ldots \ldots \ldots$ & 1994 Oct 19 & $22: 05: 33$ & 645.422 & 100 & 5 \\
\hline $52624 \ldots \ldots \ldots$ & 1994 Oct 23 & $21: 50: 52$ & 649.410 & 82 & 4 \\
\hline $52625 \ldots \ldots \ldots$ & 1994 Oct 23 & $23: 54: 31$ & 649.496 & 55 & 2 \\
\hline $52675 \ldots \ldots \ldots$ & 1994 Oct 27 & $21: 54: 02$ & 653.414 & 70 & 3 \\
\hline $52676 \ldots \ldots \ldots$ & 1994 Oct 27 & $23: 47: 07$ & 653.492 & 55 & 2 \\
\hline $52702 \ldots \ldots \ldots$ & 1994 Oct 31 & $21: 29: 18$ & 657.395 & 70 & 3 \\
\hline $52703 \ldots \ldots \ldots$ & 1994 Oct 31 & $23: 28: 26$ & 657.477 & 45 & 2 \\
\hline $52724 \ldots \ldots \ldots$ & 1994 Nov 04 & $14: 50: 23$ & 661.117 & 90 & 4 \\
\hline $52762 \ldots \ldots \ldots$ & 1994 Nov 08 & $16: 37: 19$ & 665.191 & 110 & 5 \\
\hline $52790 \ldots \ldots \ldots$ & 1994 Nov 12 & $16: 36: 56$ & 669.191 & 110 & 5 \\
\hline $52820 \ldots \ldots \ldots$ & 1994 Nov 17 & $16: 24: 58$ & 674.184 & 95 & 5 \\
\hline
\end{tabular}


TABLE $1-$ Continued

\begin{tabular}{|c|c|c|c|c|c|}
\hline $\begin{array}{c}\text { SWP } \\
\text { Image Number } \\
\text { (1) }\end{array}$ & $\begin{array}{l}\text { UT } \\
\text { Date } \\
(2)\end{array}$ & $\begin{array}{l}\text { UT Start } \\
\text { (hh:mm:ss) } \\
(3)\end{array}$ & $\begin{array}{c}\text { JD } \\
(-2,449,000) \\
(4)\end{array}$ & $\begin{array}{c}\text { Exposure Time } \\
\text { (minutes) } \\
\text { (5) }\end{array}$ & $\begin{array}{c}\text { Number of } \\
\text { Segments } \\
(6)\end{array}$ \\
\hline 52845 & 1994 Nov 20 & $16: 59: 31$ & 677.207 & 70 & 3 \\
\hline 52895 & 1994 Nov 24 & $16: 40: 42$ & 681.195 & 100 & 4 \\
\hline $52963 \ldots \ldots \ldots$ & 1994 Dec 02 & $13: 58: 18$ & 689.082 & 130 & 6 \\
\hline 52997 & 1994 Dec 06 & $14: 41: 52$ & 693.113 & 100 & 4 \\
\hline 53034 & 1994 Dec 10 & $14: 24: 25$ & 697.102 & 100 & 5 \\
\hline 53053 & 1994 Dec 14 & $06: 36: 57$ & 700.776 & 70 & 3 \\
\hline $53106^{\mathrm{a}} \ldots \ldots \ldots \ldots$ & 1994 Dec 17 & $14: 35: 48$ & 704.108 & 90 & 4 \\
\hline $53160^{\mathrm{a}}$. & 1994 Dec 22 & $15: 18: 53$ & 709.138 & 20 & 1 \\
\hline $53191 \ldots$ & 1994 Dec 26 & $14: 38: 53$ & 713.109 & 75 & 4 \\
\hline
\end{tabular}

${ }^{a}$ Image dropped because the target was not properly centered within the aperture.

${ }^{b}$ Exposure guided on offset star in the FES field.

tion (about 8 months) used in the original NGC 5548 and NGC 3783 IUE campaigns in order to facilitate a more direct comparison with these lower luminosity Seyfert galaxies. The observations taken during this campaign are described in $\S 2$, the data are presented in $\S 3$, and the results are discussed in $\S 4$. Finally, the main conclusions are summarized in $\S 5$.

\section{OBSERVATIONS}

From 1994 April 28 to December $26(\Delta T=242$ days $)$, Fairall 9 was observed with $I U E$ at nearly regular intervals of about 4 days. All observations were made through the large aperture $\left(10^{\prime \prime} \times 20^{\prime \prime}\right)$ of the short wavelength spectrograph (1150-1950 $\AA$ ) in the low-dispersion mode $(\sim 6 \AA)$. During the 63 different observing epochs of $4 \mathrm{hr}$ (occasionally $8 \mathrm{hr}$ ) each, a total of 80 spectra were recorded with the short wavelength prime (SWP) camera (Harris \& Sonneborn 1987).

The fine error sensor (FES) anomaly, which is attributed to scattered light entering the IUE telescope tube, has not been reported to contaminate any SWP image, since it was found for the first time in 1991 (Weinstein \& Carini 1992). However, the solar-like spectrum of this scattered light affects the FES, a photomultiplier with a bandpass from $3900 \AA$ to $9000 \AA$ (S-20 cathode), with a sensitivity strongly peaked at $4600 \AA$ (Holm \& Rice 1981). The FES is used for target acquisition and fine guiding during exposures; hence, these operations are significantly influenced by the presence of the scattered light. For most IUE observations of Fairall 9, there was no star within the FES field of view suitable for tracking. In these cases, the total integration time was achieved by adding up a number of shorter exposures, typically 15-30 minutes long. After each of these segments, the $I U E$ telescope was moved to point to a nearby bright star to check the pointing stability.

As a consequence of the scattered light, the photometric capabilities of the FES could not be used to obtain a simultaneous optical light curve for Fairall 9. However, an optical light curve has been obtained from the nearly simultaneous ground-based optical campaign, and these data will be presented separately (Santos-Lleó et al. 1997).

The log of the IUE observations (Table 1) includes, for each SWP image number (col. [1]), the UT date (col. [2]), time (col. [3]), and Julian Date (col. [4]) of the start of the exposure, the total integration time (col. [5]), and the number of segments in which this integration was achieved (col. [6]).

\section{DATA ANALYSIS AND RESULTS}

All the images have been processed with the new IUE
Final Archive processing software, NEWSIPS (Nichols et al. 1993). The major advantage of this software is the method of the raw science data registration, which significantly reduces the fixed pattern noise in the IUE images and improves the photometric correction. NEWSIPS also includes a weighted slit extraction method (Kinney, Bohlin, \& Neill 1991) and rederived absolute flux calibrations (González-Riestra, Cassatella, \& de la Fuente 1992). All of these improvements result in higher photometric accuracy and higher signal-to-noise ratio spectra, when compared to the data processed with the old software (IUESIPS; Turnrose \& Thompson 1984).

Because of the acquisition and tracking problems mentioned in $\S 2$, we have checked every image to identify those in which Fairall 9 may have gone out of the aperture for some time during the total exposure. A first test was made by checking the FES recentering errors after those exposures that were not guided; a benchmark test made with an $I U E$ standard star has shown that up to \pm 25 units in the $X$-axis and \pm 7 units in the $Y$-axis can be accepted as tolerance limits to ensure that the target did remain in the aperture (Rodríguez-Pascual 1997). Another check is based on the position within the IUE SWP camera at which the spectrum was recorded. In the spatially resolved spectrum (SILO file of NEWSIPS processing software), we have measured the shift in the spatial direction with respect to the center of the aperture. The wavelength of the peak of the strongest emission lines (Ly $\alpha$ and $\mathrm{C}$ IV 21550 ) has been used as reference for the shift in the spectral direction. The 12 spectra recorded more than 4 pixels away from the expected position in either spatial or spectral directions have been rejected and are not considered in this paper.

\subsection{Continuum and Emission-Line Measurements}

As shown in Figure 1, the continuum flux density has been measured in four bands that are free of obvious emission or absorption features in the mean spectrum: 1380 $1400 \AA, 1500-1520 \AA, 1780-1810 \AA$, and $1860-1900 \AA$ (observed wavelengths). The corresponding flux densities with their associated uncertainties are listed in Table 2 and plotted in Figure 2. Note that the integration times have been adjusted to reach an optimal exposure in the peaks of the strongest emission lines ( $\operatorname{Ly} \alpha, \mathrm{C}$ IV $\lambda 1550)$ at the cost of underexposing the continuum, where the flux density is 5 times weaker; this results in relatively large uncertainties in the continuum flux-density measurements.

With regard to the emission lines, no attempt has been made to deconvolve the observed profiles into components. Consequently, the Ly $\alpha$ flux includes the flux from the $\mathrm{N} \mathrm{v}$ $\lambda 1240$ line as well, and we will refer to the measured flux as 


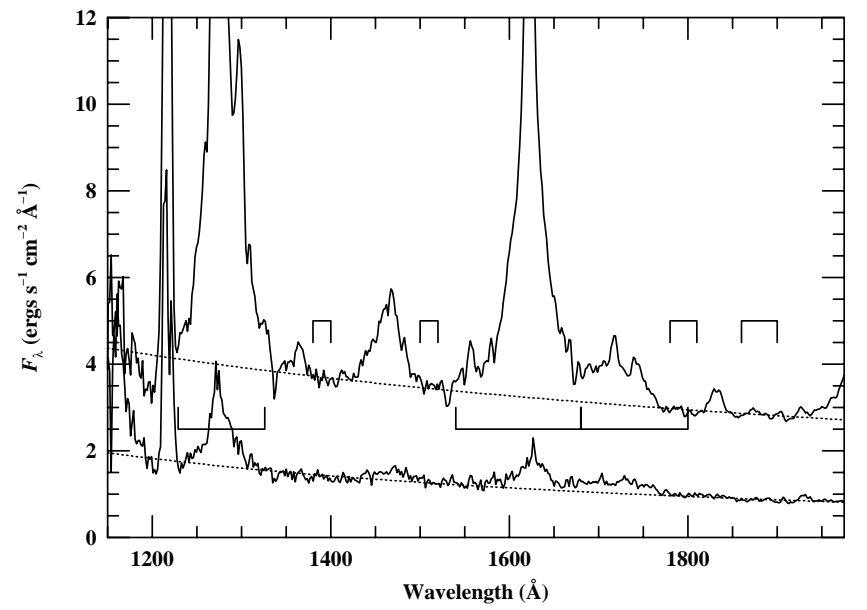

FIG. 1.-Mean (top) and rms (bottom) SWP spectra of Fairall 9. The bands in which the continuum has been measured are marked above the spectrum, and the dotted lines show power-law fits to the fluxes in these bands in both the mean and rms spectra. The Ly $\alpha+\mathrm{N} v, \mathrm{C}$ IV $\lambda 1550$, and $\mathrm{He}$ II $\lambda 1640$ integration ranges are marked below the mean spectrum.

Ly $\alpha+\mathrm{N}$ v. The measurements of $\mathrm{C}$ IV $\lambda 1550$ and $\mathrm{He}$ II $\lambda 1640$ are to some extent $(<2 \%)$ affected by the overlap of the red wing of $\mathrm{C}$ IV $\lambda 1550$ with the blue wing of $\mathrm{He}$ II $\lambda 1640$. The observed wavelength ranges used for integration of the Ly $\alpha+\mathrm{N}$ v, Si IV $\lambda 1400, \mathrm{C}$ IV $\lambda 1550$, and He II $\lambda 1640$ line fluxes are, respectively, $1229-1326 \AA, 1400-1500 \AA, 1540-$ $1680 \AA$, and $1680-1800 \AA$, as marked in Figure 1.

The continuum underneath the emission lines has been estimated by linear interpolation between the four continuum bands described above. The absorption lines have not

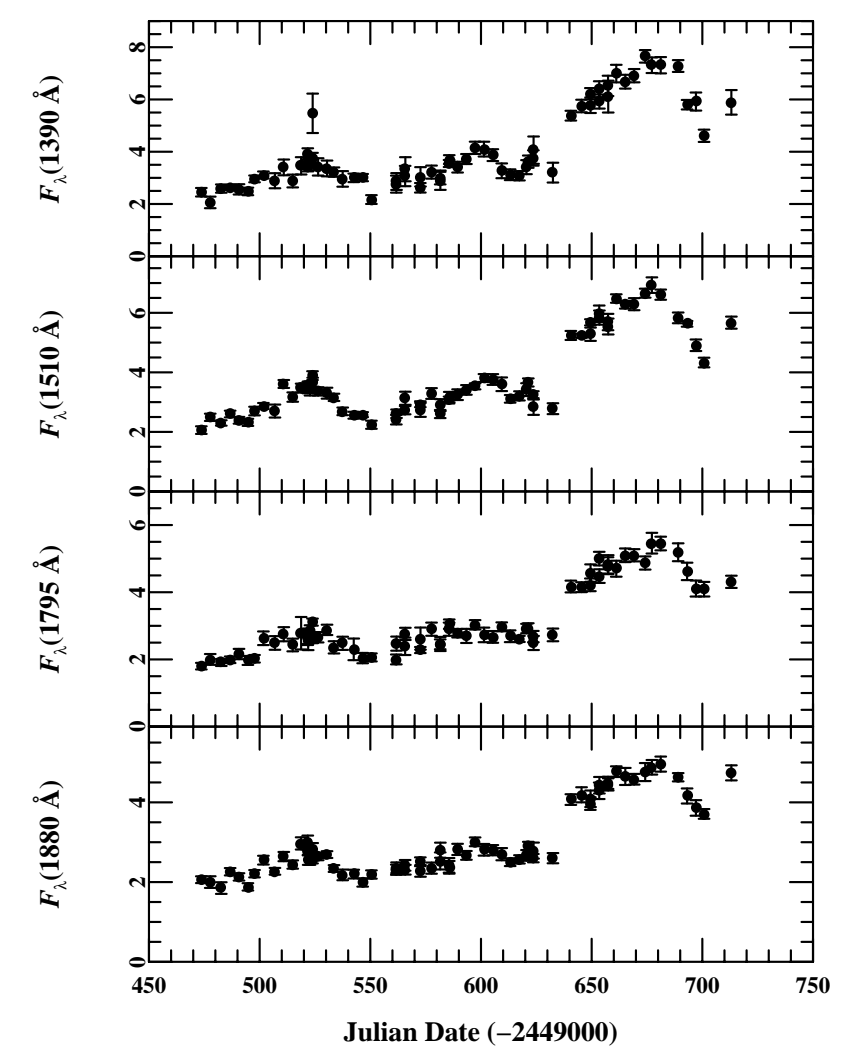

FIG. 2.-Light curves of the continuum fluxes at $1390 \AA$, $1510 \AA$, 1795 $\AA$, and $1880 \AA$ Å (observed wavelengths).
TABLE 2

CONTINUUM FLUXeS

\begin{tabular}{|c|c|c|c|c|}
\hline $\begin{array}{c}\text { Julian Date } \\
(-2,449,000) \\
(1)\end{array}$ & $\begin{array}{c}F_{\lambda}(1390 \AA)^{\mathrm{a}} \\
(2)\end{array}$ & $\begin{array}{c}F_{\lambda}(1510 \AA)^{\mathrm{a}} \\
(3)\end{array}$ & $\begin{array}{c}F_{\lambda}(1795 \AA)^{\mathrm{a}} \\
\quad(4)\end{array}$ & $F_{\lambda}(1880 \AA)^{\mathrm{a}}$ \\
\hline 1 & 6 & 13 & \pm 0.10 & $2.06 \pm 0.09$ \\
\hline 477.684 . & & & & 01 \\
\hline & & & & \\
\hline 76. & & $0 \pm 0.11$ & & \\
\hline & & & & \\
\hline & & & & $88 \pm 0.09$ \\
\hline & $2.96 \pm 0.13$ & & & \\
\hline$J$ & J & & & \\
\hline & 9 & 22 & & $7 \pm 0.09$ \\
\hline & $3.40 \pm$ & & & \\
\hline & & & & \\
\hline & $3.46 \pm$ & & & 15 \\
\hline & & & & \\
\hline & & & & \\
\hline & & & & \\
\hline & & & & \\
\hline & & & & \\
\hline & & & & \\
\hline & & & & \\
\hline & & & & \\
\hline & & & & \\
\hline & & & & \\
\hline & & & & \\
\hline & & & & \\
\hline & & & & \\
\hline & & & & \\
\hline & & & & \\
\hline & & & & \\
\hline & & & & \\
\hline & & & & \\
\hline & & & & \\
\hline & & & & \\
\hline & & & & \\
\hline & & & & \\
\hline & & & & \\
\hline & & & & \\
\hline & & & & \\
\hline & & & & \\
\hline & & & & \\
\hline & & & & \\
\hline & & & & \\
\hline & & & & \\
\hline & & & & \\
\hline & & & & \\
\hline & & & & \\
\hline & & 4 & & \\
\hline & & & & \\
\hline & & & & \\
\hline & & & & \\
\hline & & 5 & & \\
\hline & & & & \\
\hline & & & & \\
\hline & & & & \\
\hline & & & & \\
\hline & & & & \\
\hline & & & & \\
\hline & & & & \\
\hline & & & & \\
\hline & & 3 & & \\
\hline & & 20 & 18 & \\
\hline & & & & \\
\hline & & & & \\
\hline & & & & \\
\hline & & 5. & $5.19 \pm$ & \\
\hline & & & & \\
\hline & & & & \\
\hline & & & & \\
\hline 713.109 . & $5.89 \pm 0.47$ & $5.67 \pm 0.20$ & $4.31 \pm 0.18$ & $4.74 \pm 0.19$ \\
\hline
\end{tabular}

${ }^{a}$ Units are $10^{-14} \mathrm{ergs} \mathrm{cm}^{-2} \mathrm{~s}^{-1} \AA^{-1}$. 
been removed from the emission-line fluxes, since the strongest absorption features (C II, $\mathrm{Si}$ II, $\mathrm{C}$ IV) seen in the average spectrum are difficult to identify even in the bestexposed single spectrum. The fluxes and their associated uncertainties are listed in Table 3, and the emission-line light curves are shown in Figure 3.

Initially, the error estimates for the line and continuum measurements are based on the errors assigned by the NEWSIPS extraction software to the individual pixels in the relevant integration range for each feature. However, it became clear from the resulting light curves that the uncertainties estimated in this fashion are too large. We will make the assumption that the relative magnitudes of the errors obtained in the initial estimate are correct, but that all of the uncertainties should be scaled down by a constant multiplicative factor.

The uncertainties in the line and continuum fluxes can be estimated by measurements closely spaced in time, where differences should be attributable primarily to random errors rather than intrinsic variations. We consider the ratio of two measurements of the same flux $f_{i}$ and $f_{j}$ that are separated by some short time interval $\Delta t_{i j}=t_{i}-t_{j}$. For $\Delta t_{i j}$ sufficiently small, $f_{i}$ and $f_{j}$ are essentially independent measurements of the same quantity, and thus the uncertainties in their ratio ought to be $\sigma_{i j}^{2} \approx \sigma_{i}^{2}+\sigma_{j}^{2}$, where $\sigma_{n}$ is the uncertainty associated with measurement $f_{n}$. Averaged over many pairs of observations, the variance of the distribution of $f_{i} / f_{j}$ will be $\sigma_{i j}^{2} /\left\langle f^{2}\right\rangle$, where $\left\langle f^{2}\right\rangle$ is the mean square flux, provided that there is no intrinsic variability on timescales shorter than the largest value of $\Delta t_{i j}$. Thus, for closely spaced points (i.e., those with $\Delta t_{i j}$ less than some small

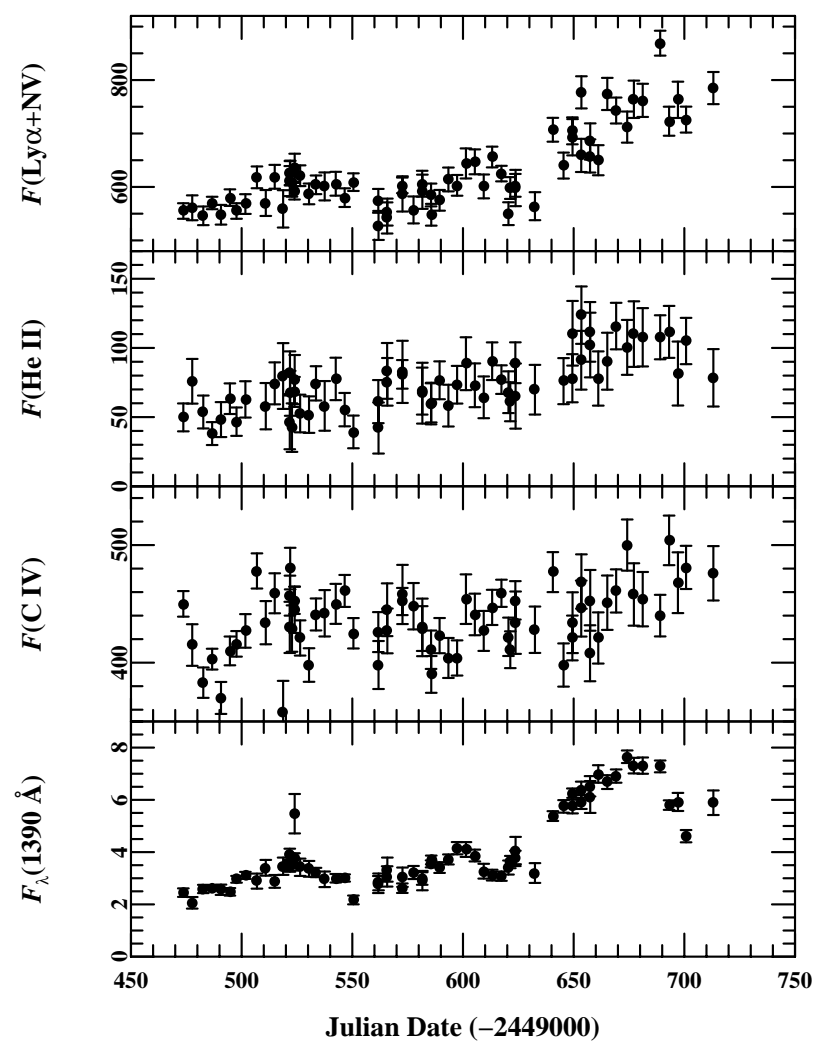

FIG. 3.-Light curves of the UV emission lines in which significant variability was detected. The bottom panel shows the $1390 \AA$ A continuum light curve for easy comparison.
TABLE 3

EMISSION-LiNe FLUXES

\begin{tabular}{|c|c|c|c|}
\hline $\begin{array}{c}\text { Julian Date } \\
(-2,449,000) \\
(1)\end{array}$ & $\begin{array}{c}\text { Ly } \alpha \lambda 1215^{\mathrm{a}} \\
\text { (2) }\end{array}$ & $\begin{array}{c}\mathrm{C} \text { IV } \lambda 1550^{\mathrm{a}} \\
\text { (3) }\end{array}$ & $\begin{array}{c}\text { He II } \lambda 1640^{\mathrm{a}} \\
\text { (4) }\end{array}$ \\
\hline $473.652 .$. & $555 \pm 15$ & $450 \pm 11$ & $49.8 \pm 10.1$ \\
\hline $477.684 \ldots \ldots$ & $561 \pm 23$ & $415 \pm 18$ & $75.9 \pm 16.2$ \\
\hline $482.672 \ldots \ldots$ & $546 \pm 17$ & $383 \pm 13$ & $53.7 \pm 11.9$ \\
\hline 486.676 & $570 \pm 12$ & $403 \pm 9$ & $38.1 \pm 8.3$ \\
\hline $490.676 \ldots$ & $548 \pm 18$ & $370 \pm 14$ & $48.2 \pm 12.6$ \\
\hline $494.695 \ldots \ldots$ & $580 \pm 16$ & $410 \pm 12$ & $63.3 \pm 11.1$ \\
\hline $497.688 \ldots \ldots$ & $555 \pm 15$ & $416 \pm 11$ & $46.7 \pm 10.2$ \\
\hline $501.691 \ldots \ldots$ & $568 \pm 18$ & $427 \pm 14$ & $62.8 \pm 13.1$ \\
\hline 506.918 . & $618 \pm 20$ & $478 \pm 15$ & \\
\hline $510.910 \ldots \ldots$ & $570 \pm 24$ & $434 \pm 18$ & $57.9 \pm 16.7$ \\
\hline $514.941 \ldots \ldots$ & $619 \pm 22$ & $459 \pm 17$ & $74.4 \pm 15.2$ \\
\hline $518.746 \ldots \ldots$ & $559 \pm 35$ & $358 \pm 27$ & $79.7 \pm 23.8$ \\
\hline $521.746 \ldots \ldots$ & $611 \pm 28$ & $430 \pm 22$ & $46.2 \pm 19.4$ \\
\hline $521.836 \ldots \ldots$ & $626 \pm 22$ & $457 \pm 17$ & $82.1 \pm 15.4$ \\
\hline $521.930 \ldots \ldots$ & $626 \pm 23$ & $480 \pm 18$ & $67.2 \pm 16.2$ \\
\hline $522.750 \ldots \ldots$ & $607 \pm 25$ & $429 \pm 20$ & $42.4 \pm 17.5$ \\
\hline $523.879 \ldots \ldots$ & $592 \pm 16$ & $453 \pm 12$ & $68.2 \pm 10.8$ \\
\hline $523.961 \ldots \ldots$ & $636 \pm 26$ & $445 \pm 20$ & $77.1 \pm 17.8$ \\
\hline $526.434 \ldots \ldots$ & $621 \pm 19$ & $421 \pm 15$ & $52.6 \pm 13.6$ \\
\hline $530.441 \ldots \ldots$ & $587 \pm 19$ & $398 \pm 14$ & $51.8 \pm 13.2$ \\
\hline $533.590 \ldots \ldots$ & $604+17$ & $441+14$ & $74.4+12.4$ \\
\hline $537.520 \ldots \ldots$ & $601 \pm 26$ & $442 \pm 20$ & $58.1 \pm 18.0$ \\
\hline $542.516 \ldots \ldots$ & $606 \pm 22$ & $450 \pm 17$ & $77.6 \pm 15.3$ \\
\hline $546.543 \ldots \ldots$ & $580 \pm 17$ & $461 \pm 14$ & $55.4 \pm 12.1$ \\
\hline $550.496 \ldots \ldots$ & $609 \pm 16$ & $425 \pm 13$ & $39.3 \pm 11.8$ \\
\hline $561.746 \ldots \ldots$ & $574 \pm 22$ & $426 \pm 17$ & $61.2 \pm 15.6$ \\
\hline $561.840 \ldots \ldots$ & $528 \pm 27$ & $398 \pm 20$ & $42.1 \pm 18.5$ \\
\hline $565.734 \ldots \ldots$ & $542 \pm 29$ & $445 \pm 22$ & $83.4 \pm 20.2$ \\
\hline $565.824 \ldots \ldots$ & $553 \pm 25$ & $427 \pm 19$ & $75.5 \pm 17.2$ \\
\hline $572.590 \ldots \ldots$ & $603+15$ & $452+12$ & $80.9+10.3$ \\
\hline $572.664 \ldots \ldots$ & $587 \pm 33$ & $458 \pm 25$ & $82.7 \pm 22.5$ \\
\hline $577.656 \ldots \ldots$ & $557+25$ & $448+20$ & \\
\hline $581.574 \ldots \ldots$ & $606 \pm 24$ & $429 \pm 19$ & $68.8 \pm 17.0$ \\
\hline $581.648 \ldots \ldots$ & $591 \pm 32$ & $430 \pm 24$ & $67.3 \pm 22.0$ \\
\hline $585.574 \ldots$ & $585 \pm 21$ & $411 \pm 16$ & $59.6 \pm 14.8$ \\
\hline $585.660 \ldots \ldots$ & $548 \pm 20$ & $390 \pm 16$ & $60.5 \pm 14.4$ \\
\hline $589.422 \ldots \ldots$ & $575 \pm 19$ & $423 \pm 15$ & $76.6 \pm 13.7$ \\
\hline $593.410 \ldots \ldots$ & $614 \pm 22$ & $404 \pm 17$ & $58.3 \pm 15.1$ \\
\hline $597.340 \ldots \ldots$ & $603+19$ & $404+15$ & $73.4+13.6$ \\
\hline $601.336 \ldots$ & $644 \pm 28$ & $454 \pm 21$ & $88.6 \pm 19.1$ \\
\hline $605.352 \ldots \ldots$ & $647+23$ & $441+18$ & $73.0+15.8$ \\
\hline $609.367 \ldots \ldots$ & $601 \pm 22$ & $427 \pm 17$ & $64.3 \pm 15.1$ \\
\hline $613.172 \ldots \ldots$ & $656 \pm 19$ & $447 \pm 15$ & $90.4 \pm 13.6$ \\
\hline $617.367 \ldots$ & $625 \pm 15$ & $459 \pm 12$ & $77.1 \pm 10.3$ \\
\hline $620.543 \ldots \ldots$ & $550 \pm 21$ & $422 \pm 16$ & $68.0 \pm 15.1$ \\
\hline $621.367 \ldots \ldots$ & $598 \pm 20$ & $411 \pm 16$ & $61.1 \pm 14.1$ \\
\hline $623.656 \ldots \ldots$ & $603 \pm 21$ & $453 \pm 16$ & $89.3 \pm 14.8$ \\
\hline $623.758 \ldots \ldots$ & $598 \pm 34$ & $434 \pm 27$ & $65.2 \pm 23.5$ \\
\hline $632.508 \ldots \ldots$ & $564 \pm 26$ & $428 \pm 20$ & $69.8 \pm 18.0$ \\
\hline $640.594 \ldots \ldots$ & $707+22$ & $477+17$ & \\
\hline $645.422 \ldots \ldots$ & $640 \pm 24$ & $398 \pm 18$ & $76.0 \pm 16.6$ \\
\hline $649.410 \ldots \ldots$ & $705+25$ & $421+19$ & $78.1+17.4$ \\
\hline $649.496 \ldots$ & $693 \pm 34$ & $434 \pm 26$ & $110.6 \pm 23.4$ \\
\hline $653.414 \ldots \ldots$ & $659 \pm 31$ & $446 \pm 24$ & $91.1 \pm 21.2$ \\
\hline $653.492 \ldots \ldots$ & $777 \pm 30$ & $469 \pm 23$ & $123.7 \pm 20.7$ \\
\hline $657.395 \ldots$ & $658 \pm 31$ & $408 \pm 24$ & $111.6 \pm 21.6$ \\
\hline $657.477 \ldots$ & $685 \pm 34$ & $453 \pm 26$ & $102.1 \pm 23.4$ \\
\hline $661.117 \ldots$. & $650 \pm 28$ & $421 \pm 22$ & $77.9 \pm 19.6$ \\
\hline $665.191 \ldots \ldots$ & $774 \pm 30$ & $451 \pm 23$ & $90.4 \pm 20.6$ \\
\hline $669.191 \ldots \ldots$ & $743 \pm 24$ & $461 \pm 18$ & $115.8 \pm 16.8$ \\
\hline $674.184 \ldots \ldots$ & $712 \pm 29$ & $500 \pm 22$ & $100.4 \pm 19.8$ \\
\hline $677.207 \ldots$ & $764 \pm 35$ & $458 \pm 27$ & $110.0 \pm 23.7$ \\
\hline $681.195 \ldots \ldots$ & $762 \pm 31$ & $454 \pm 23$ & $107.7 \pm 21.1$ \\
\hline $689.082 \ldots \ldots$ & $869 \pm 23$ & $440 \pm 18$ & $107.7 \pm 15.9$ \\
\hline $693.113 \ldots$. & $723 \pm 27$ & $504 \pm 21$ & $111.6 \pm 18.8$ \\
\hline $697.102 \ldots$. & $763 \pm 34$ & $468 \pm 26$ & $81.5 \pm 23.1$ \\
\hline $700.776 \ldots \ldots$ & $726 \pm 24$ & $481 \pm 18$ & $105.0 \pm 16.7$ \\
\hline $713.109 \ldots \ldots$ & $785 \pm 30$ & $476 \pm 23$ & $78.4 \pm 20.7$ \\
\hline
\end{tabular}

${ }^{\mathrm{a}}$ Units are $10^{-14} \mathrm{ergs} \mathrm{cm}^{-2} \mathrm{~s}^{-1}$. 
value), we can compute the variance of the distribution in $f_{i} / f_{j}$ from the actual flux measurements and compare this directly with the mean value of $\sigma_{i j}^{2} /\left\langle f^{2}\right\rangle$ computed from the error estimates. We find that the root mean square (rms) of the $f_{i} / f_{j}$ distribution is in all cases smaller than expected from the quoted error estimates and thus reduce all of the error estimates by a constant multiplicative factor (which ranges from 1.03 in the case of $L y \alpha+\mathrm{N} v$ to 3.4 for the 1510 $\AA$ continuum) to bring the two error estimates into agreement. This is a conservative approach: if there is intrinsic variability on short timescales, then the rms of the $f_{i} / f_{j}$ distribution ought to be larger than the mean value of $\sigma_{i j}^{2} /\left\langle f^{2}\right\rangle$, contrary to what is found. The true uncertainties may be even smaller than the rescaled error estimates, since any low-level variability that occurs on short timescales is attributed to random error in this estimate. Operationally, the factors we use for rescaling the NEWSIPS uncertainties are determined by considering pairs of observations separated by less than 1 day. We also find that if we increase the maximum time interval to 4 days, which greatly increases the number of pairs of observations in the calculation, then the rescaling factors increase (as expected), but only slightly, indicating that there is little intrinsic variability on such short timescales.

The uncertainties quoted in Tables 2 and 3 and illustrated in the figures are the rescaled values computed as described.

\subsection{Variability}

The variability parameters of the continuum and emission lines are given in Table 4 . The entries in Table 4 are the unweighted mean flux $\langle f\rangle$ (col. [2]) and rms flux $\sigma$ (col. [3]) for the entire campaign. These are defined in the usual way, i.e., for $N$ individual observations $f_{i}$ the mean is

$$
\langle f\rangle=\frac{1}{N} \sum_{i=1}^{N} f_{i}
$$

and $\sigma$ is the square root of the variance

$$
\sigma^{2}=\frac{1}{N-1} \sum_{i=1}^{N}\left(f_{i}-\langle f\rangle\right)^{2} .
$$

Also in Table 4 are two common measures of the variability $F_{\text {var }}$ (col. [4]) and $R_{\max }$ (col. [5]). The quantity $R_{\max }$ is simply the ratio of maximum to minimum flux. The quantity $F_{\mathrm{var}}$ is an estimate of the rms of the intrinsic variability relative to the mean flux, i.e., corrected for the effect of

TABLE 4

\begin{tabular}{|c|c|c|c|c|}
\hline $\begin{array}{l}\text { Feature } \\
\text { (1) }\end{array}$ & $\begin{array}{l}\text { Mean Flux } \\
\text { (2) }\end{array}$ & $\begin{array}{l}\text { Standard } \\
\text { Deviation }^{\mathrm{a}} \\
\text { (3) }\end{array}$ & $\begin{array}{c}F_{\text {var }} \\
(4)\end{array}$ & $\begin{array}{c}R_{\max } \\
(5)\end{array}$ \\
\hline 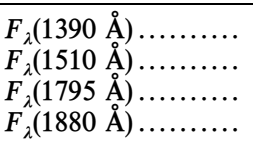 & $\begin{array}{l}4.11 \\
3.82 \\
3.14 \\
3.02\end{array}$ & $\begin{array}{l}1.54 \\
1.35 \\
1.04 \\
0.92\end{array}$ & $\begin{array}{l}0.367 \\
0.350 \\
0.325 \\
0.300\end{array}$ & $\begin{array}{l}3.72 \pm 0.42 \\
3.36 \pm 0.24 \\
3.03 \pm 0.24 \\
2.68 \pm 0.24\end{array}$ \\
\hline 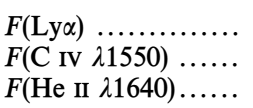 & $\begin{array}{r}628 \\
436 \\
75\end{array}$ & $\begin{array}{l}73 \\
29 \\
21\end{array}$ & $\begin{array}{l}0.110 \\
0.049 \\
0.155\end{array}$ & $\begin{array}{l}1.65 \pm 0.10 \\
1.41 \pm 0.12 \\
3.25 \pm 0.89\end{array}$ \\
\hline
\end{tabular}

VARIABILITY PARAMETERS

${ }^{a}$ Units are $10^{-14}$ ergs cm ${ }^{-2} \mathrm{~s}^{-1} \AA^{-1}$ for continuum fluxes and $10^{-14}$ ergs $\mathrm{cm}^{-2} \mathrm{~s}^{-1}$ for emission lines. random errors, i.e.,

$$
F_{\text {var }}=\frac{\left(\sigma^{2}-\Delta^{2}\right)^{1 / 2}}{\langle f\rangle}
$$

where the quantity $\Delta^{2}$ is the mean square value of the uncertainties $\Delta_{i}$ associated with the fluxes $f_{i}$, i.e.,

$$
\Delta^{2}=\frac{1}{N} \sum_{i=1}^{N} \Delta_{i}^{2}
$$

As found in previous studies of other AGNs (Clavel et al. 1991; Reichert et al. 1994; Korista et al. 1995; Crenshaw et al. 1996), the amplitude of the continuum variations in Fairall 9 decreases at longer UV wavelengths. Whether this is due to an intrinsic hardening of the featureless continuum as the source becomes more luminous or to the presence of an intrinsically less variable feature, whose contribution increases with wavelength, cannot be addressed with the limited wavelength range covered by the SWP camera. However, based on the analysis of IUE archival data obtained between 1979 to 1991 and published optical data, Recondo-González et al. (1997) conclude that the Balmer continuum and the pseudocontinuum formed by blended emission lines of $\mathrm{Fe}$ II contribute significantly to the continuum flux in the UV range covered by the SWP camera. For a continuum flux similar to that observed during the 1994 campaign, their estimates of the combined contribution of Balmer continuum and $\mathrm{Fe}$ II lines to the bands centered at $1795 \AA$ and $1880 \AA$ are 0.56 and $0.51 \times 10^{-14} \mathrm{ergs} \mathrm{cm}^{-2}$ $\mathrm{s}^{-1} \AA^{-1}$, respectively. Subtraction of these values from the mean flux densities in Table 4 results in all the continuum bands varying with the same amplitude $\left(F_{\text {var }}=0.34 \pm 0.03\right)$.

Another test that can be done to determine whether or not the AGN continuum hardens as it becomes brighter is to compare on a linear plot simultaneously measured fluxes in two well-separated wave bands. In the case of NGC 5548, for example, a plot of the optical flux (at $5100 \AA$ ) versus the short-wavelength UV flux (at $1350 \AA ̊$ ) shows (1) that the best fit to these requires some curvature, and (2) that the optical axis intercept is nonzero (Fig. 3 of Peterson 1991). The sense of the curvature in this relationship means that the amplitude of the UV variations is greater than the amplitude of the optical variations, i.e., the variable part of the spectrum becomes harder as the continuum gets brighter. The nonzero intercept of the optical axis gives a measure of the constant starlight contribution to the optical fluxes, which in this case is in good agreement with the estimate obtained by image decomposition (Romanishin et al. 1995).

In Figure 4, we plot $F_{\lambda}(1880 \AA)$ versus $F_{\lambda}(1390 \AA)$ to test for curvature that would indicate a nonlinear relationship between these two continuum bands. A linear fit to these data (as shown in Fig. 4) provides a good fit $\left(\chi_{v}^{2}=3.08\right)$; indeed, a quadratic gives a slightly worse fit $\left(\chi_{v}^{2}=3.11\right)$ and the curvature coefficient is statistically insignificant. We conclude that, unlike the case of NGC 5548, and as previously concluded on the basis of existing data on this object, in the case of Fairall 9 there is no evidence that the AGN continuum shape changes as the luminosity changes. To test more directly the hypothesis that the relationship between the two continuum bands is linear, we fit the data to the equation

$$
\log F_{\lambda}(1880 \AA)=C+\gamma \log F_{\lambda}(1390 \AA),
$$




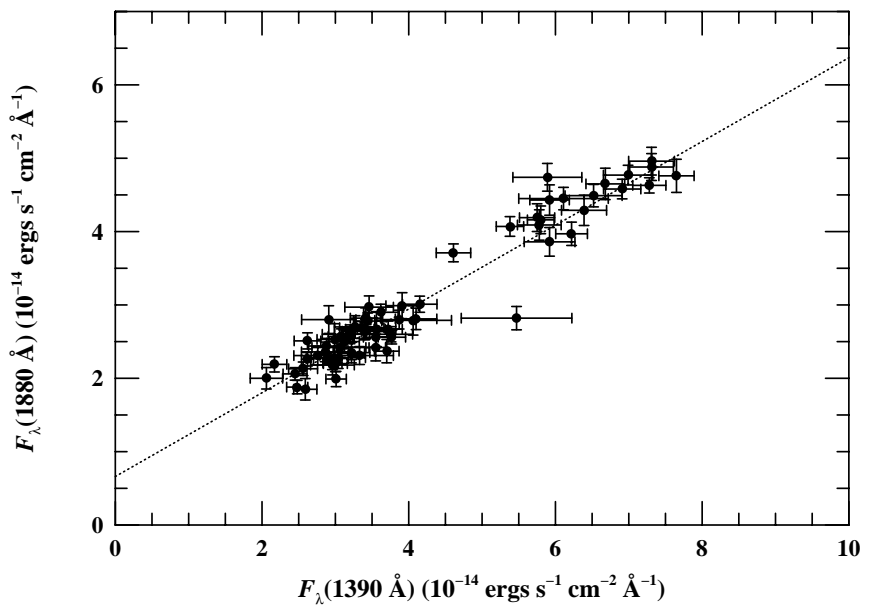

Fig. 4.-Simultaneous measurements of the continuum flux at $1390 \AA$ and $1880 \AA$, from the data in Table 2. The dotted line shows the best linear fit to these data. There is no evidence for curvature in this relationship, which would indicate that the spectral index of the continuum depends on luminosity.

where $C$ is a constant, which we assume to be the $y$ intercept of Figure 4 (i.e., $C=6.6 \times 10^{-15} \mathrm{ergs} \mathrm{s}^{-1} \mathrm{~cm}^{-2}$ $\AA^{-1}$ ). This yields $\gamma=0.992 \pm 0.031$, consistent with a linear relationship. This will be tested more critically over a wider wavelength range using the combined UV and optical data on this object (Santos-Lleó et al. 1997).

Among the emission lines, the largest variations occur in Ly $\alpha+\mathrm{N}$ V and He II $\lambda 1640$, and C IV $\lambda 1550$ varies only weakly. The light curves we measure for Si IV $\lambda 1400$ show no statistically significant variability, and therefore we do not include these measurements in the tables or figures. We must point out, however, that the $\mathrm{Si}$ IV $\lambda 1400$ feature appears, albeit weakly, in the rms spectrum shown in Figure 1. Weak variations did in fact occur, but the measured light curve is dominated by random noise.

The continuum light curves show three "events" of similar duration ( $\sim 70$ days), but with noticeably different amplitudes (Fig. 2). The first two events show variations of up to about $50 \%$, while during the last event the continuum varied by more than a factor of 2 . The $L y \alpha+N$ v light curve is qualitatively similar to that of the continuum, with two low-amplitude events $(\sim 20 \%)$ followed by a stronger $(\sim 50 \%)$ third one. The most remarkable difference is that the fading of the continuum by the end of the campaign is not clearly seen in the $\mathrm{Ly} \alpha+\mathrm{N} v$ light curve. Although still smaller than that of the continuum, the amplitude of the He II $\lambda 1640$ variations is the largest among the emission lines. The detection of the first two events in the He II $\lambda 1640$ and $C$ IV $\lambda 1550$ light curves is quite marginal, but the onset of the third event is clear even in these noisy light curves.

\subsection{Cross-Correlation Analysis}

The emission-line time delays, or lags, relative to the continuum variations can be quantified by cross-correlation of the continuum and emission-line light curves. Before computing the cross-correlation functions (CCFs), multiple measurements obtained during single observing shifts were averaged, weighted in each case by the inverse square of their assigned uncertainties. The CCFs have been computed by using three different algorithms: (1) the interpolation cross-correlation function (ICCF) of Gaskell \& Sparke
(1986), (2) the discrete correlation function (DCF) method of Edelson \& Krolik (1988), and (3) the $z$-transformed discrete correlation function (ZDCF) method of Alexander (1996). The ICCF and DCF algorithms are as described by White \& Peterson (1994). All three methods give similar, although not identical, results. The CCFs between the continuum at $1390 \AA$ and the other continuum bands (Fig. 5) show that they are all highly correlated, with no measurable delay among the different bands. In Table 5 we summarize the characteristics of the CCFs, where $\tau_{\text {peak }}$ is the location of the ICCF peak, which has value $r_{\text {max }} ; \tau_{\text {cent }}$ is the centroid for ICCF values greater than $0.8 r_{\max }$; and FWHM is the full width of the ICCF at $0.5 r_{\text {max }}$. Also in Table 5 are the ZDCF results; $\tau_{\mathrm{ML}}$ is the maximum likelihood estimate of the location of the true peak in the $\mathrm{CCF}$, and the errors associated with it represent a $68 \%$ confidence interval (see Alexander 1996 and Edelson et al. 1996). The maximum likelihood uncertainties quoted in Table 5 are more conservative error estimates than we obtain using either the analytic formula of Gaskell \& Peterson (1987), which yields estimated errors $\Delta \tau \approx 4-7$ days for all ICCFs computed in this paper, or error estimates based on Monte Carlo simulations (e.g., White \& Peterson 1994), which yield $\Delta \tau \lesssim 2$ days for all ICCFs presented here.

The results of cross-correlating the continuum at $1390 \AA$ and the three emission-line light curves of Table 3 and Figure 3 are shown in Figure 6 and summarized in Table 5. Only Ly $\alpha+\mathrm{N} v$ and He II $\lambda 1640$ are highly correlated with the continuum $\left(r_{\max }>0.8\right)$. For $\mathrm{C}$ IV $\lambda 1550, r_{\max }=0.63$ is statistically significant (for $N=55$ epochs) at greater than

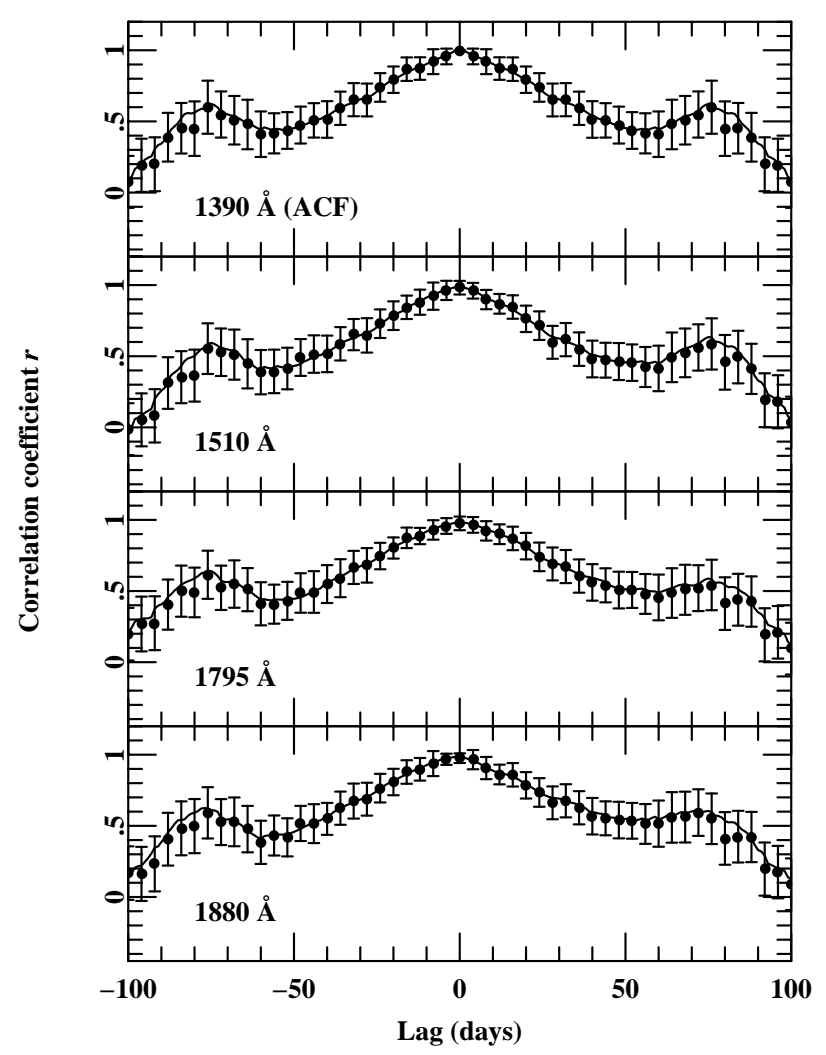

FIG. 5.-Continuum cross-correlation functions (CCFs). The panels show the CCFs (ICCF as solid lines; DCF as individual points with associated error bars) for the $1390 \AA$ continuum and (a) itself (i.e., the ACF), (b) the $1510 \AA$ continuum, (c) the $1795 \AA$ continuum, and (d) the $1880 \AA$ continuum. 
TABLE 5

Cross-Correlation Results

\begin{tabular}{|c|c|c|c|c|c|c|c|}
\hline \multirow[b]{2}{*}{ Feature } & \multicolumn{4}{|c|}{$\mathrm{ICCF} / \mathrm{DCF}$} & \multicolumn{3}{|c|}{$\mathrm{ZDCF}$} \\
\hline & $r_{\max }$ & $\begin{array}{c}\text { FWHM } \\
\text { (days) }\end{array}$ & $\begin{array}{c}\tau_{\text {peak }} \\
\text { (days) }\end{array}$ & $\begin{array}{c}\tau_{\text {cent }} \\
\text { (days) }\end{array}$ & $r_{\max }$ & $\begin{array}{c}\tau_{\mathrm{ML}} \\
\text { (days) }\end{array}$ & $\begin{array}{c}\tau_{\text {cent }} \\
\text { (days) }\end{array}$ \\
\hline $1390 \AA \AA_{0}(\mathrm{ACF}) \ldots \ldots$ & 1.00 & 88 & 0 & 0.0 & & & \\
\hline $1510 \AA \ldots \ldots \ldots \ldots \ldots$ & 0.98 & 87 & -1 & -0.5 & 0.94 & $3.7_{-7.0}^{+1.9}$ & -0.4 \\
\hline $1795 \AA \ldots \ldots \ldots \ldots$ & 0.98 & 104 & 1 & 0.0 & 0.93 & $3.7_{-7.6}^{+3.2}$ & 1.6 \\
\hline $1880 \AA ̊ \ldots \ldots \ldots \ldots$ & 0.98 & 131 & -2 & -1.0 & 0.94 & $-3.9_{-2.3}^{+7.2}$ & 7.8 \\
\hline Ly $\alpha \lambda 1215 \ldots \ldots \ldots$. & 0.91 & 106 & 14 & 14.6 & 0.86 & $3.6_{-2.2}^{+12.0}$ & 19.5 \\
\hline $\mathrm{C}$ IV $\lambda 1550 \ldots \ldots \ldots$ & 0.63 & 75 & $34::$ & $31.5::$ & 0.51 & $43.8_{-24.5}^{+25.4}$ & 29.7 \\
\hline He II $\lambda 1640 \ldots \ldots \ldots$ & 0.85 & 145 & 10 & 3.7 & 0.63 & $-3.9_{-9.7}^{+17.4}$ & 4.2 \\
\hline
\end{tabular}

99.9\% confidence. However, the CCF is very flat-topped and the width of the CCF is somewhat smaller than that of the continuum autocorrelation function (ACF). We therefore attach no significance to the measured $\mathrm{C}$ IV $\lambda 1550 \mathrm{lag}$.

\subsection{Line Profile Variations}

The low amplitude of the line variations limits the depth of the analysis of the profile variability. However, inspection of Figures 2 and 3 shows that, during this observing campaign, Fairall 9 went through two different levels of brightness, and a simple comparison can be made of the profiles in the high and low states. For the high state, we have averaged all the spectra from JD 2,449,660 to the end of the campaign, and for the low state, the spectra taken between JD 2,449,530 and JD 2,449,580 have been averaged. The Ly $\alpha, \mathrm{C}$ IV $\lambda 1550$, and He II $\lambda 1640$ line profiles for the two

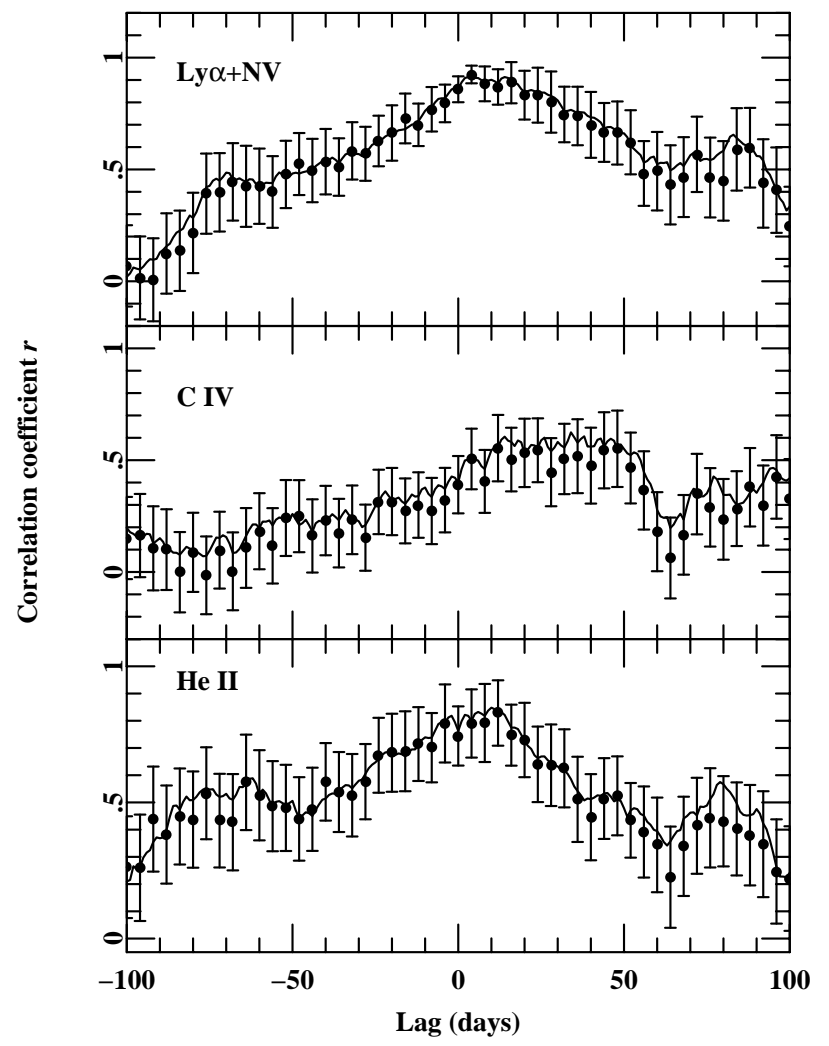

FIG. 6.-Continuum-emission-line CCFs. The panels show the CCFs (ICCF as solid lines; DCF as individual points with associated error bars) for the $1390 \AA$ continuum and (top) Ly $\alpha+\mathrm{N}$ v, (middle) C IV $\lambda 1550$, and (bottom) He II $\lambda 1640$. states are shown in Figure 7, after continuum subtraction. Although the error bars are rather large, the difference profile of C IV $\lambda 1550$ appears to be narrower than the Ly $\alpha$ and He II $\lambda 1640$ difference profiles. In an attempt to quantify roughly the changes in the line profiles, each difference profile has been fitted with a Gaussian (shown as a thick line in the bottom panels of Fig. 7). We find that the width of the C Iv $\lambda 1550$ difference profile $\left(4180 \pm 340 \mathrm{~km} \mathrm{~s}^{-1}\right)$ is less than half the width of the $\operatorname{Ly} \alpha\left(10600 \pm 460 \mathrm{~km} \mathrm{~s}^{-1}\right)$ and $\mathrm{He}$ II $\lambda 1640\left(13890 \pm 900 \mathrm{~km} \mathrm{~s}^{-1}\right)$ difference profiles. Also, the center of the Gaussian fitted to $C$ IV $\lambda 1550$ is more redshifted relative to the galaxy redshift $(c \Delta z=1570 \pm 170$ $\left.\mathrm{km} \mathrm{s}^{-1}\right)$ than are either Ly $\alpha\left(880 \pm 130 \mathrm{~km} \mathrm{~s}^{-1}\right)$ or $\mathrm{He}$ II $\lambda 1640\left(270 \pm 400 \mathrm{~km} \mathrm{~s}^{-1}\right)$. However, given the low level of emission-line variability detected in this experiment, we are reluctant to draw strong conclusions based on these limited data. We draw attention to these difference because they are of potential importance should they be substantiated by better data.

\section{DISCUSSION}

The selection of Fairall 9 for an intensive monitoring effort with $I U E$ was intended to extend the very limited sample of intensively monitored AGNs to higher luminosity and thus test critically the predicted BLR radius-luminosity relationship $R \propto L^{1 / 2}$. A previous effort by Clavel et al. (1989) (see also Koratkar \& Gaskell 1989; Lub \& de Ruiter 1992; Shields, Ferland, \& Peterson 1995; RecondoGonzález et al. 1997) yielded lags of about $115 \pm 70$ days for $L y \alpha$ and about $200 \pm 80$ days for $C$ IV $\lambda 1550$. These lags were measured during a factor of 30 decrease in luminosity and are based on data that are not well sampled on short timescales. Indeed, cross-correlation lags are strongly influenced by inflection points in light curves, and the previously published Fairall 9 light curves are not well sampled at the time the continuum and emission lines passed through their respective minima. Moreover, a plot of the observed C IV $\lambda 1550$ time lag versus UV continuum for reasonably well observed AGNs (e.g., Fig. 3 of Peterson 1994) suggests that the published emission-line time lags for Fairall 9 are somewhat larger than would be expected by extrapolation from the lower luminosity Seyfert galaxies, assuming either the predicted $R \propto L^{1 / 2}$ or the possibly shallower luminosity dependence weakly suggested by the other data. For example, if we scale the NGC 5548 results $^{44}$ (Clavel et al.

\footnotetext{
${ }^{44}$ The values of $\tau_{\text {cent }}$ in NGC 5548 are about 11 days for both lines, using the interpolation CCF as described by White \& Peterson (1994).
} 

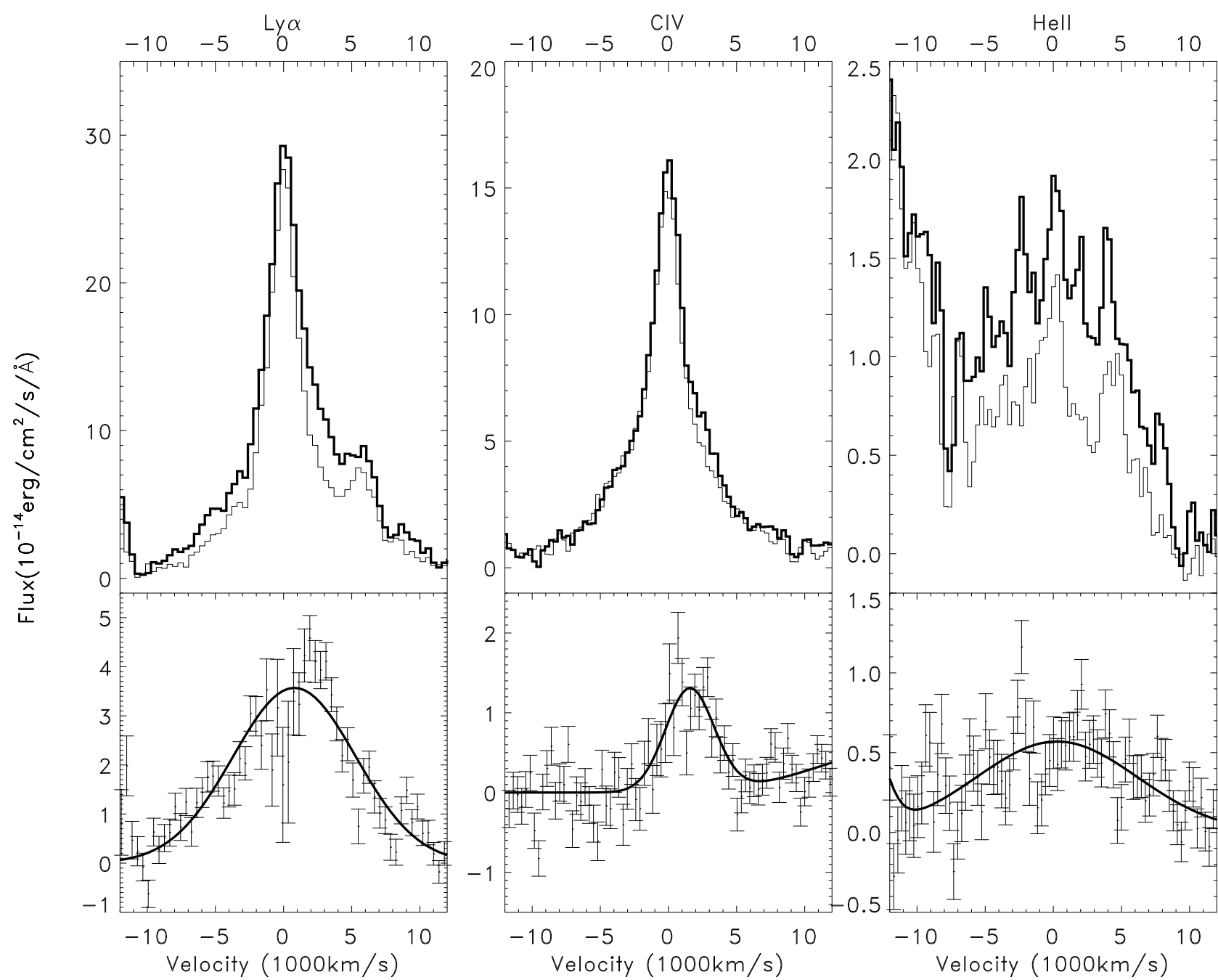

Fig. 7.- The average profiles at high (thick lines) and low (thin lines) states (top) and their differences (bottom) are shown for Ly $\alpha$ (left), C IV $\lambda 1550$ (middle), and He II $\lambda 1640$ (right). Note that the overlap in the profiles of C IV $\lambda 1550$ and He II $\lambda 1640$ is not present in their difference profiles. The thick lines in the bottom panels show the Gaussian fits to the difference profiles.

1991) assuming the $L^{1 / 2}$ luminosity dependence, then we would expect an Ly $\alpha \mathrm{C}$ iv $\lambda 1550$ lag of between 13 and 73 days for Fairall 9 for its low and high historical states, respectively. These considerations led us specifically to look for more rapid BLR response than could have been detected in the original Clavel et al. (1989) experiment.

For Ly $\alpha+\mathrm{N} v$ we find $\tau_{\text {cent }} \approx 14-20$ days for this experiment, which is not much smaller than the value of 28 days we would expect by scaling the NGC 5548 results to the mean luminosity of Fairall 9 during this campaign. Unfortunately, relatively weak variability during the first several months of the campaign precluded an accurate measurement of the $\mathrm{C}$ IV $\lambda 1550$ response time. Formally, we obtain $\tau_{\text {cent }} \approx 30$ days for $C$ IV 21550 , although we place no confidence in this measurement on account of the low signal-tonoise ratio of the $\mathrm{C}$ IV $\lambda 1550$ light curve.

The reason for the dramatic difference between the Clavel et al. (1989) $\operatorname{Ly} \alpha+\mathrm{N} v$ lag and that reported here is not obvious. Just as the previous data were not sensitive to short-timescale emission-line response, the present campaign was not sensitive to long-timescale $(R / c \gtrsim 100$ days $)$ emission-line lags. Among likely possibilities are the following:
1. The BLR did not change between the two experiments. On account of their very different sampling characteristics, the two monitoring programs sampled completely different scales in a very extended BLR. The lack of an obvious turnover in the Ly $\alpha+\mathrm{N} v$ light curve at the end of the campaign may be consistent with this interpretation.

2. As a consequence of the significant change in mean luminosity between the two campaigns (a factor of $\sim 3-6$, depending on how it is measured), there has been a change in the optical depth in BLR clouds in the sense that the more recent experiment samples clouds that are currently optically thick (and thus produced highly variable emission lines), but which were optically thin (and producing less variable emission lines) in the previous campaign. Such dramatic changes are possible on account of the short recombination time for high-density BLR clouds, $\tau_{\text {rec }} \approx$ $10^{9} / n_{\mathrm{e}}\left(\mathrm{cm}^{-3}\right) \mathrm{hr}$. This could occur if much of the line response we see in this campaign originates in clouds that have column densities only somewhat deeper than the ionized column; if the continuum flux increased by a significant factor, then these clouds would be completely ionized and thus show no recombination-line variability with further increases in continuum flux. Similar clouds at larger 
distances would remain partially neutral and responsive to continuum changes.

3. There has been a significant rearrangement of the BLR gas between the two experiments. The crossing time for an emission-line cloud in the BLR is $\tau_{\mathrm{dyn}} \approx c \tau_{\text {cent }} / \Delta V$, where $\Delta V$ is the emission-line width. Taking $\Delta V=10^{4} \mathrm{~km} \mathrm{~s}^{-1}$ and $\tau_{\text {cent }} \approx 150$ days yields $\tau_{\text {dyn }} \approx 10 \mathrm{yr}$, which is approximately the interval of time between the observations reported here and those made as Fairall 9 passed through the minimum state recorded by Clavel et al. (1989); it is certainly plausible that a significant rearrangement of the BLR gas has occurred between the two experiments.

Detailed modeling beyond the scope of this paper will be required to support further or to eliminate these and other possibilities. Unfortunately, a longer campaign that might have discriminated among the various possibilities is simply not possible on account of the termination of IUE operations.

The ICCF and DCF indicate that He II $\lambda 1640$, with $\tau_{\text {cent }} \lesssim 4$ days, responds more rapidly than does $\operatorname{Ly} \alpha+\mathrm{N} \mathrm{v}$, which is consistent with what has been found in other sources and is consistent with a radially ionization-stratified BLR. However, the uncertainties in all of these measurements are sufficiently large that we cannot rule out the possibility that $\mathrm{Ly} \alpha+\mathrm{N}$ v and $\mathrm{He}$ II $\lambda 1640$ actually respond with the same lag.

The C IV $\lambda 1550$ results, however, remain puzzling. In other well-studied AGNs, the response times and line profiles for $L y \alpha$ and C IV $\lambda 1550$ are similar. While these data do not provide a strong constraint on the $C$ IV $\lambda 1550$ response time, the variable part of the C IV $\lambda 1550$ line profile appears to be significantly narrower and redshifted relative to the Ly $\alpha$ and He II $\lambda 1640$ lines. It appears to be rather doubtful that the peak response of $\mathrm{C}$ IV $\lambda 1550$ and Ly $\alpha$ occurs in the same gas, as might be more easily inferred for NGC 5548, for example.

Given (1) the possible similarity of the $\operatorname{Ly} \alpha+\mathrm{N}$ v and $\mathrm{He}$ II $\lambda 1640$ responses, (2) our expectation from other experiments that Ly $\alpha$ and C IV $\lambda 1550$ ought to respond on the same timescale, and (3) some, albeit unreliable, indications that the $\mathrm{C}$ IV $\lambda 1550$ response is longer than $\operatorname{Ly} \alpha+\mathrm{N} \mathrm{v}$ in Fairall 9, we might well ask if the response we are seeing in $\mathrm{Ly} \alpha$ is in fact not $\mathrm{Ly} \alpha$ at all, but $\mathrm{N} v \lambda 1240$, which, again based on other sources, we expect to respond like He II 21640. The contribution of the $\mathrm{N} v \lambda 1240$ emission line to the Ly $\alpha+\mathrm{N} v$ light curve may cause the relatively short lag found if the variations in this high-ionization line are sufficiently large. We have considered this explanation and reject it for the following reasons. First, there is no structure in the difference profile shown in Figure 7 that may suggest that $\mathrm{N} v \lambda 1240$ significantly contributes to the variations. Second, N v $\lambda 1240$ appears only weakly in the rms spectrum (Fig. 1), again arguing that the amplitude of $\mathrm{N} v \lambda 1240$ is insufficient to contribute in a significant way to the Ly $\alpha+\mathrm{N}$ v variability. Third, as a final check, we remeasured the Ly $\alpha$ flux as before, but we extended the measurement only to $1228 \AA$ (in the rest frame) to minimize contamination by $\mathrm{N} v \lambda 1240$; this yielded Ly $\alpha$ light curves and CCFs that did not differ in any substantive way from the $\mathrm{Ly} \alpha+\mathrm{N} v$ results reported here.

\section{SUMMARY}

The results of the IUE monitoring campaign on a bright Seyfert 1 nucleus (Fairall 9) qualitatively support the general conclusions drawn from previous campaigns on the less luminous AGNs NGC 3783, NGC 4151, and NGC 5548. In particular, the various continuum bands respond simultaneously, within our ability to measure them, and the emission lines respond to continuum variations on timescales that are very short (i.e., days), although the relatively noisy emission-line light curves measured in this experiment result in large uncertainties in the emission-line time lags. The principal conclusions of this study are as follows:

1. Large-amplitude (factor of 2) UV continuum variations occur in Fairall 9 on a timescale ( $~ 70$ days) similar to the fastest variations detected (at a similar amplitude) in NGC 5548, which is about 6 times less luminous.

2. No significant lags have been found among the four UV continuum bands measured, although the amplitude of the variations decreases with increasing wavelength. Unlike NGC 5548, we do not find evidence that the AGN continuum changes shape (i.e., spectral index) as the luminosity changes. The wavelength baseline over which this has been tested will be extended to the optical region in a forthcoming paper (Santos-Lleó et al. 1997).

3. Among the emission lines, He II $\lambda 1640$ shows the largest fractional variations. The variations in $\operatorname{Ly} \alpha+\mathrm{N} \mathrm{v}$ and $\mathrm{C}$ IV $\lambda 1550$ are much smaller.

4. The lags $\left(\tau_{\text {cent }}\right)$ between the emission lines and the UV continuum are $\lesssim 4$ days for He II $\lambda 1640$ and about $14-20$ days for $\operatorname{Ly} \alpha+\mathrm{N} v$. The uncertainties of these determinations are large ( $\sim 5-10$ days), so it cannot be concluded with any confidence that the lags for these two lines are not the same, although this is much clearer in other sources. The small variations in C IV $\lambda 1550$ make it difficult to estimate a lag, but it seems to be greater than for $\operatorname{Ly} \alpha+\mathrm{N} \mathrm{v}$ and $\mathrm{He}$ II $\lambda 1640$.

5. The line profile variations in Ly $\alpha$ and $\mathrm{He}$ II $\lambda 1640$ differ from those in C IV $\lambda 1550$. The variable part of the C IV $\lambda 1550$ line appears to be narrower and more redshifted than the variable parts of Ly $\alpha$ and $\mathrm{He}$ II $\lambda 1640$.

This work has been supported in part by NASA through grant NAG 5-3233 to Ohio State University.

\section{REFERENCES}

Alexander, T. 1996, MNRAS, in press

Alloin, D., Clavel, J., Peterson, B. M., Reichert, G. A., \& Stirpe, G. M. 1994,

in Frontiers of Space and Ground-based Astronomy, ed. W. Wamsteker,

M. S. Longair, \& Y. Kondo (Dordrecht: Kluwer), 423

Alloin, D., et al. 1995, A\&A, 293, 293

Blandford, R. D., \& McKee, C. F. 1982, ApJ, 255, 419

Clavel, J., et al. 1991, ApJ, 366, 64 (Paper I)

Clavel, J., Wamsteker, W., \& Glass, I. S. 1989, ApJ, 337, 236

Crenshaw, D. M., et al. 1996, ApJ, 470, 322

Dietrich, M., et al. 1993, ApJ, 408, 416 (Paper IV)

Edelson, R. A., et al. 1996, ApJ, 470, 364
Edelson, R. A., \& Krolik, J. H. 1988, ApJ, 333, 646

Gaskell, C. M., \& Peterson, B. M. 1987, ApJS, 65, 1

Gaskell, C. M., \& Sparke, L. S. 1986, ApJ, 305, 175

Gondhalekar, P. M., Horne, K., \& Peterson, B. M., eds. 1994, ASP Conf. Proc. 69, Reverberation Mapping of the Broad-Line Region in Active Galactic Nuclei (San Francisco: ASP)

González-Riestra, R., Cassatella, A., \& de la Fuente, A. 1992, Record of the IUE Three Agency Coordination Meeting (Madrid)

Harris, A. W., \& Sonneborn, G. 1987, in Exploring the Universe with the IUE Satellite, ed. Y. Kondo (Dordrecht: Kluwer), 729

Holm, A. V., \& Rice, G. 1981, NASA IUE Newsl., 15, 74 
Kaspi, S., et al. 1996, ApJ, 470, 336

Kinney, A. L., Bohlin, R. C., \& Neill, J. D. 1991, PASP, 103, 694

Koratkar, A. P., \& Gaskell, C. M. 1989, ApJ, 345, 637

Korista, K. T., et al. 1995, ApJS, 97, 285 (Paper VIII)

Lub, J., \& de Ruiter, H. R. 1992, A\&A, 256, 33

Maoz, D., et al. 1993, ApJ, 404, 576

Nichols, J. S., Garhart, M. P., De la Peña, M. D., \& Levay, K. L. 1993 NEWSIPS Information Manual: Low Dispersion Data, Version 1.0 (CSC/SD-93/6062)

Peterson, B. M. 1991, in Variability of Active Galaxies, ed. W. J. Duschl, S. J. Wagner, \& M. Camenzind (Berlin: Springer), 47 1993, PASP, 105, 247

. 1994, in ASP Conf. Proc. 69, Reverberation Mapping of the Broad-Line Region in Active Galactic Nuclei, ed. P. M. Gondhalekar, K. Horne, \& B. M. Peterson (San Franciso: ASP), 1

Peterson, B. M., et al. 1991, ApJ, 368, 119 (Paper II)
Peterson, B. M., et al. 1992, ApJ, 392, 470 (Paper III) 1994, ApJ, 425, 622 (Paper VII)

Recondo-González, M. C., et al. 1997, A\&AS, 121, 461

Reichert, G. A., et al. 1994, ApJ, 425, 582 (Paper V)

Rodríguez-Pascual, P. M. 1997, ESA IUE Newsl., in press

Romanishin, W., et al. 1995, ApJ, 455, 516

Santos-Lleó, M., et al. 1997, in preparation

Shields, J. C., Ferland, G. J., \& Peterson, B. M. 1995, ApJ, 441, 507

Stirpe, G. M., et al. 1994, ApJ, 425, 609

Turnrose, B. E., \& Thompson, R. W. 1984, International Ultraviolet Explorer Image Processing Information Manual, Version 2.0 (CSC/TM84/6058)

Warwick, R., et al. 1996, ApJ, 470, 349

Weinstein, D., \& Carini, M. 1992, NASA IUE Newsl., 48, 143

White, R. J., \& Peterson, B. M. 1994, PASP, 106, 879 